\title{
Hat die Psychoonkologie die Onkologie verändert?
}

\author{
30 Jahre Psychoonkologie in der Schweiz, zehn Jahre Schweizerische Gesellschaft für \\ Psychoonkologie: Die SGPO nahm diese Jubiläen zum Anlass, um im Rahmen einer \\ nationalen Fachtagung Rückblick zu halten, Themen zu vertiefen und künftige Heraus- \\ forderungen für die Entwicklung einer hochwertigen Psychoonkologie zu benennen.
}

Ursula Rohrera,

Diana Zwahlen ${ }^{b}$

a Assistenzpsychologin, BSc, Onkologie, Universitätsspital Basel

b Psychologin, OnkologiePsychosomatik, Universitätsspital Basel
Korrespondenz:

Dr. phil. Diana Zwahlen Universitätsspital Basel Onkologie

Petersgraben 4

CH-3041 Basel

diana.zwahlen[at]usb.ch
Über zwei Tage erstreckte sich die Jubiläumstagung, zu der die Schweizerische Gesellschaft für Psychoonkologie (SGPO) im Juni nach St. Gallen geladen hatte. 220 Teilnehmerinnen und Teilnehmer waren ins Hotel Einstein gekommen, um die zahlreichen Referate und Workshops zu aktuellen Themen aus der Psychoonkologie zu besuchen. Referenten aus der Schweiz, Deutschland und Österreich bereicherten das Programm.

Es sei ein grosser Erfolg, dass es der einst kleinen SGPO gelungen ist, heute eine solch vielfältige Tagung auf die Beine zu stellen, sprach Dr. Brigitta Wössmer, Präsidentin der SGPO, in ihrem Grusswort den mittlerweile 200 Mitgliedern der Gesellschaft ihre Wertschätzung aus. «Wir dürfen uns aber nicht ausruhen», machte sie weiter klar. Nach der erfolgreichen Etablierung der interprofessionellen Weiterbildung in Psychoonkologie gelte es nun, die Implementierung nationaler Guidelines für die Psychoonkologie und die Finanzierung psychoonkologischer Leistungen sicherzustellen. Die Antwort auf die Leitfrage der Tagung nahm Brigitta Wössmer vorweg: Ja, die Psychoonkologie habe die Onkologie verändert - und zwar positiv.

Als prominenter Gast würdigte auch Heidi Hanselmann, Regierungsrätin und Vorsteherin des St. Galler Gesundheitsdepartementes, die Psychoonkologie als festen Bestandteil der Onkologie, die standhaft darin bleibe, stets den Patienten in den Mittelpunkt zu stellen. «Wenn es die SGPO nicht schon gäbe, müsste man sie erfinden und zwar subito», unterstrich die Politikerin und wünschte der noch jungen Gesellschaft Motivation, Wachstum und Anerkennung auf ihrem weiteren Weg.

Das letzte Jahrhundert liess Prof. Jakob Passweg, Präsident der Krebsliga Schweiz, im Schnelldurchlauf Revue passieren. Der Kampf gegen den Krebs sei vielfach mit kriegerischem Vokabular geführt worden. Dass heute eine andere Sprache gepflegt werde, sei der Psychoonkologie zu verdanken. Sie habe ihm die Bedeutung der Kommunikation gelehrt.

\section{Fokuserweiterung: von der Lebensdauer bis hin zur Lebensqualität}

Auch Prof. Thomas Cerny, Präsident der Stiftung Krebsforschung, lud die Tagungsteilnehmenden zu einer Reise zurück in die Pionierzeit der Onkologie ein, als
Krebs noch als soziales Stigma galt und Präsident Nixon zum «War on Cancer» aufrief. Explizit strich er den Berner Medizinprofessor Rolf H. Adler und sein Buch «Psychosomatik als Wissenschaft» heraus, läuteten diese doch den Beginn einer neuen Sichtweise ein, die in der Schweiz früher als im benachbarten Nachkriegs-Europa ihren Platz finden konnte. Der grosse Verdienst der Psychoonkologie sei es, dass das Augenmerk heute nicht mehr nur auf die Lebensdauer, sondern auch auf die Lebensqualität von Krebsbetroffenen gerichtet werde.

Nach dem packenden Rückblick fokussierte Prof. Anja Mehnert, Leiterin der Sektion Psychosoziale Onkologie am Universitätsklinikum Leipzig, auf die Gegenwart. Sie wies auf einen zunehmenden Zeitdruck bei Ärzten und die erhöhte Belastung des Pflegepersonals hin. Es bestehe eine Diskrepanz zwischen der zunehmend technologisierten Medizin und dem Bedürfnis der Betroffenen nach menschlicher Unterstützung. «Es gibt keine Zeit um zuzuhören», betonte die Psychologin und erinnerte daran, dass die Folgen von nicht erfülltem Unterstützungsbedarf eine höhere Anzahl an Symptomen, Konsultationen und mehr gesundheitsschädigendes Verhalten seien. In der Psychoonkologie gehe es darum, sich an den Bedürfnissen der Patienten zu orientieren. Denn «am Ende des Lebens sind wir keine Experten mehr, dann ist der Patient unser wichtigster Lehrer.»

Der Nachmittag war diversen Workshops gewidmet. So konnten die Teilnehmenden Themen wie Achtsamkeit, Komplementärmedizin, Umgang mit dysfunktionalen familiären Mustern oder Fragen zur Akzeptanz der Psychoonkologie in der Onkologie vertiefen. Im Symposium zu «Speziellen Gruppen» gaben Dr. Stefan Essig vom Institut für Sozial- und Präventivmedizin an der Universität Bern und Prof. Alexander Kiss, Chefarzt Psychosomatik, Universitätsspital Basel, gemeinsam einen spannenden Einblick in die Arbeit mit Adoleszenten und die Schwierigkeiten in der Kommunikation zwischen Jugendlichen und Ärzten sowie Pflegenden. Adoleszente stellen eine wenig untersuchte Patientengruppe dar, die durch den Konflikt zwischen anstehender Ablösung und dennoch benötigter Unterstützung zusätzlich belastet sind. Stefan Essig, der nicht nur Mediziner, sondern auch Betroffener ist, griff das Thema Fertilität auf, das im Gespräch mit Jugendlichen oft zu wenig thematisiert wird. 
Im Fokus des Referates von Elisabeth Andritsch, Universitätsklinik Graz, standen Kinder krebskranker Eltern. Sie unterstrich die Bedeutung des Coachings von Eltern, die mit der Kommunikation und dem Umgang mit der Krankheit in der Familie oft überfordert seien. Dr. Christoph Hürny von der Geriatrischen Klinik St. Gallen berichtete über die psychosozialen Aspekte bei älteren und alten Menschen mit Krebs. Er erinnerte daran, dass diese grosse Patientengruppe dem Tempo im medizinischen Umfeld nicht immer gewachsen sei und mehr Rücksichtnahme auf ihre speziellen Bedürfnisse brauche. Zum Thema Depressionen gab er zu bedenken, dass sich diese bei älteren Menschen häufig in Körpersymptomen äusserten.

\section{SGPO-Präsidium für Judith Alder, Forschungspreis für Corina Rüegg}

Erstmals wurde an einer SGPO-Tagung ein Wissenschaftssymposium durchgeführt. Sechs junge Wissenschaftlerinnen präsentierten ihre Forschungsarbeiten einer dreiköpfigen Fachjury. Im Rahmen der festlichen Abendveranstaltung konnte dann Corina Rüegg, PhD-Studentin am Institut für Sozial- und Präventivmedizin, Bern, den mit 1500 Franken dotierten Forschungspreis für ihre Arbeit zur Bedeutung von chronischen Gesundheitsproblemen für die Lebensqualität von jungen Erwachsenen, die in der Kindheit an Krebs erkrankt waren, entgegennehmen. Einen weiteren Höhepunkt stellte die Mitgliederversammlung der SGPO dar. Dort übergab Brigitta Wössmer, die während der ersten zehn Jahre als Präsidentin die Gesellschaft umsichtig und engagiert geleitet hatte, den Stab an PD Dr. phil. Judith Alder, die einstimmig zur neuen Präsidentin gewählt wurde.

\section{Psychoonkologie unterstützt die} Vorbereitung auf die palliative Situation

Auch der zweite Tagungstag wartete mit einem breiten Programm auf. So berichtete unter anderem lic. phil. Christophe Rieder, Leitender Psychologe der Berner Klinik Montana, von den Bedürfnissen der Patienten und Patientinnen aus dem Blickwinkel der stationären Rehabilitation. Hinsichtlich der Passung

Aktuelle

Forumthemen

Diskutieren Sie mit Im Forum präsentieren wir regelmässig brisante Themen aus Politik, Ökonomie und Wissenschaft, die das Schweizer Gesundheitswesen betreffen. Bringen Sie Ihre Meinung ein oder kommentieren Sie die Äusserungen Ihrer Kolleginnen und Kollegen. Das Forum finden Sie unter: www.saez.ch/forum/ zwischen Ansprüchen und Potential kann für die Patienten die Anpassung der Ansprüche hilfreich sein. Die stationäre Rehabilitation und die Psychoonkologie können dabei helfen, Veränderungen in diese Richtung einzuleiten.

Auf reges Interesse stiess das Thema Komplementärmedizin. Dr. Marc Schläppi, Leiter des Zentrums für integrative Medizin am Kantonsspital St. Gallen, informierte über die wichtigsten Stärken und Gefahren des vieldiskutierten Behandlungsansatzes. Er vertrat die Ansicht, die Komplementärmedizin könne im Rahmen einer integrativen Behandlung auch über die Selbstwirksamkeit der Patienten einen positiven Beitrag zum Krankheitsverlauf leisten.

Dr. Roland Kunz, Chefarzt Palliative Care am Spital Affoltern, referierte über den für die Patienten oft schwierigen Übergang zur palliativen Situation. Er führte den Begriff der psychopalliativen Betreuung ein und beschrieb damit die umfassende Begleitung des Patienten und seiner Familie. Die Aufgabe der Psychoonkologie sah er hier besonders in der von medizinischem «Lärm» freien, frühzeitigen Vorbereitung der Betroffenen auf die neue Situation sowie in der Förderung der Selbstbestimmung der Patienten. Im Workshop zum selben Thema wurden diese Aspekte diskutiert. Als problematisch wurde dabei bereits die Definition der Phasen kurativer und palliativer Behandlung sowie der «end-of-life care» angesehen. Zudem diskutierten die Teilnehmenden darüber, wie stark vorhandene Strukturen auch die psychoonkologische Betreuung mitbestimmten. So bedeute der Übertritt in ein Hospiz häufig auch den Abbruch der psychoonkologischen Betreuung. Oft finde die Übergabe psychoonkologischer Informationen an die Fachpersonen im Hospiz nicht statt.

Gedanken zum interdisziplinären Arbeiten machte sich Prof. Martin Fey vom Inselspital Bern. Neben der psychologischen Betreuung der Krebspatienten nannte er auch die Betreuung des Ärzteund Pflegeteams sowie deren Schulung in Kommunikation als wichtige Aufgabe der Psychoonkologinnen und -onkologen. Für ihn stand fest, dass die Psychoonkologie als Bestandteil der Onkologie im Team integriert sein müsse. In seinem Fazit gab er zu bedenken, dass der alleinige Blick auf das Einnahmen-Ausgaben-Verhältnis zu kurz greife. Oft würde sich Nutzen auch in stabilen Teams zeigen.

\section{Der Umgang mit schwerkranken Patienten ist eine gesellschaftliche Aufgabe}

Zum Abschluss der Tagung blickten Exponenten der Psychoonkologie und der Onkologie in die Zukunft der jungen Disziplin. Brigitte Baschung, stellvertretende Geschäftsführerin der Krebsliga Schweiz, nannte die akademische Anerkennung der interdisziplinären Weiterbildung und deren Ausweitung auf die französische und die italienische Schweiz, die Ausarbeitung nationaler Leitlinien, die Förderung der Forschung und eine fortschrittliche Gesundheitspolitik als ihre bevorzugten «Reiseziele» der SGPO.

Dr. Mark Häfner vom Spital Bülach sprach die Nachwuchsproblematik und die Sicherstellung und Verteilung der ambulanten Angebote an, während Dr. Sandra Sieber vom Spitalzentrum Oberwallis einen hoffnungsvollen Blick in die Kristallkugel wagte. Dort sah sie eine enge Zusammenarbeit zwischen Onkologen und Psychoonkologen, gut funktionierende Screening-Verfahren und eine gesicherte Finanzierung der psychoonkologischen Leistungen.

Das letzte Fachwort der Tagung gehörte Prof. Fritz Stiefel vom CHUV in Lausanne. Er rief die Tagungsteilnehmenden dazu auf, sich nicht mit dem Status quo zufriedenzugeben, sondern den Umgang mit Krankheit und mit schwerkranken Patienten als gesellschaftliche Aufgabe wahrzunehmen. 